\title{
恒温环境中 $\mathrm{ZrB}_{2}$ 氧化行为模拟
}

\author{
周述光 ${ }^{1,2}$, 国义军 ${ }^{2}$, 刘 骁 ${ }^{2}$
}

(中国空气动力研究与发展中心 1. 空气动力学国家重点实验室; 2. 计算空气动力研究所 绵阳 621000)

摘 要: 二硼化锆 $\left(\mathrm{ZrB}_{2}\right)$ 是一种应用于高超声速飞行器的新型防热材料, 近年来受到广泛关注。本研究根据 $\mathrm{ZrB}_{2}$ 在 不同温度下的氧化产物二氧化锆 $\left(\mathrm{ZrO}_{2}\right)$ 与三氧化二硼 $\left(\mathrm{B}_{2} \mathrm{O}_{3}\right)$ 的微观结构和形貌, 改进了原有的 $\mathrm{ZrB}_{2}$ 氧化唯象模型, 研究了 $\mathrm{ZrB}_{2}$ 的氧化行为, 提出了中等温度区间液态 $\mathrm{B}_{2} \mathrm{O}_{3}$ 的生成、蒸发、填充的动态平衡关系, 并考虑了孔隙出口 处的 $\mathrm{B}_{2} \mathrm{O}_{3}$ 蒸气浓度。研究结果表明: 改进后的模型能够预测低流速准静态条件下 $\mathrm{ZrB}_{2}$ 的氧化行为, 与加热炉中的 样品恒温氧化测试结果吻合良好; 孔隙对氧化过程有较大影响，在相同的温度、氧分压下，孔隙率越大，被氧化程 度越高; 在基材表面存在 $\mathrm{B}_{2} \mathrm{O}_{3}$ 液态膜的情况下, 扩散过程对氧化速率的控制被极大地降低, 材料表现出最强的抗 氧化性能。

关 键 词: 二硼化锆; 氧化行为; 孔隙率; 模拟; 液态膜

中图分类号: V45 文献标识码: A

\section{Simulation of $\mathrm{ZrB}_{2}$ Oxidation Behavior at Constant Temperature Ambient}

\author{
ZHOU Shu-Guang ${ }^{1,2}$, GUO Yi-Jun ${ }^{2}$, LIU Xiao ${ }^{2}$ \\ (1. State Key Laboratory of Aerodynamics, China Aerodynamics Research and Development Center, Mianyang 621000, China; \\ 2. Computational Aerodynamics Institute, China Aerodynamics Research and Development Center, Mianyang 621000, China)
}

\begin{abstract}
Zirconium diboride $\left(\mathrm{ZrB}_{2}\right)$, a thermal protection material for hypersonic vehicle, has received widespread attention in recent years. Here we advance an oxidation model based on oxidation products $\left(\mathrm{ZrO}_{2}\right.$ and $\left.\mathrm{B}_{2} \mathrm{O}_{3}\right)$ and their morphology at different temperatures to simulate the oxidation behavior of $\mathrm{ZrB}_{2}$. This study further establishes the dynamic equilibrium among formation, evaporation and supplement of $\mathrm{B}_{2} \mathrm{O}_{3}$ whose concentration at ambient was assumed to be nonzero. Research results indicate that the advanced model can predict the oxidation behavior of $\mathrm{ZrB}_{2}$ under quasi-static low flow conditions and the simulation results are consistent with the data obtained from sample suspended in wire heater furnace at constant temperature. The porosity has a great influence on the oxidation process, and at the same temperature and oxygen partial pressure, the larger the porosity, the higher the oxidation degree. The oxidation rate controlled by diffusion in the presence of liquid $\mathrm{B}_{2} \mathrm{O}_{3}$ film laying outer surface of substrate reduces greatly and the material exhibits the strongest oxidation resistance.
\end{abstract}

Key words: zirconium diboride; oxidation behavior; pore fraction; simulation; film

近年来, 随着临近空间高超声速飞行器的快速 发展, 迫切需要发展新型防热材料, 或对传统热防
护材料进行升级改造，以满足热防护材料长时间气 动加热而又需维持外形的要求。二嗍化锆 $\left(\mathrm{ZrB}_{2}\right)$ 因

收稿日期: 2018-08-28; 收到修改稿日期：2018-11-23

基金项目: 国家重点基础研究发展计划(2014CB744100)

National Program on Key Basic Research Project (2014CB744100)

作者简介: 周述光(1981-), 男, 博士研究生. E-mail: zsg2015@mail.ustc.edu.cn

通讯作者：国义军，教授.E-mail: GYJ223698@sina.com 
在一定温度下烧蚀量较低，常被选作为传统碳基防 热材料的添加剂或涂层, 在高超声速飞行器热防护 系统中得到了初步应用。 $\mathrm{ZrB}_{2}$ 的研究始于 20 世纪 60 年代 ${ }^{[1]}$, 由于其在高超声速热防护系统中的应用 前景，近年来逐渐受到重视。

目前, 相关研究还处于防热材料研制和初步应用 阶段, 主要以粉体材料制备及其致密化研究为主 ${ }^{[2]}$, 对力学性能 ${ }^{[3]}$ 、抗热震性 ${ }^{[4]}$ 、抗高温氧化性能 ${ }^{[4-6]}$ 等 指标进行了优化, 而在氧化机理分析和理论建模方 面研究较少。1998 年, 赵海雷 ${ }^{[7]}$ 依据吉布斯自由能 和阿伦尼乌斯公式, 针对 $\mathrm{ZrB}_{2}$-刚玉莫来石复合材 料, 采用半试验半模型的方法, 获得了 $\mathrm{ZrB}_{2}$ 氧化质 量变化与氧化时间的关系。但该方法难于包容不同 类型试验数据, 使之满足相关建模要求。2007 年至 2009 年间, Parthasarathy ${ }^{[8-10]}$ 设定中等温度下材料外 表面气态三氧化二硼 $\left(\mathrm{B}_{2} \mathrm{O}_{3}\right)$ 产物浓度为 0 , 并假设孔 隙中 $\mathrm{B}_{2} \mathrm{O}_{3}$ 的生成速率等于其蒸发速率, 从而初步建 立了 $\mathrm{ZrB}_{2}$ 氧化模型。但该模型难以反映材料服役过 程中的变化, 相关机理分析和理论建模尚待深入。

本研究对 Parthasarathy 所建的 $\mathrm{ZrB}_{2}$ 氧化唯象模 型作了改进, 考虑了物体外表面的气态 $\mathrm{B}_{2} \mathrm{O}_{3}$ 浓度对 氧化的影响, 并舍弃了孔隙中 $\mathrm{B}_{2} \mathrm{O}_{3}$ 生成速率等于其 蒸发速率的准稳态假设, 建立了孔隙中液态 $\mathrm{B}_{2} \mathrm{O}_{3}$ 的 生成、挥发、填充三者之间的动态平衡关系。基于 该改进模型, 本研究模拟了加热炉中 $\mathrm{ZrB}_{2}$ 的氧化过 程, 分析了 $\mathrm{ZrB}_{2}$ 氧化行为的影响因素, 为深入研究 $\mathrm{ZrB}_{2}$ 材料的抗氧化性能及其潜在应用奠定了基础, 也为工程应用和材料研制中抗氧化性能评估提供了 新的方法。

\section{$1 \mathrm{ZrB}_{2}$ 氧化唯象模型框架}

在高温有氧环境中, $\mathrm{ZrB}_{2}$ 材料会发生如下系列 化学物理变化 ${ }^{[9]}$.

$$
\begin{aligned}
& \mathrm{ZrB}_{2}(\mathrm{cr})+(5 / 2) \mathrm{O}_{2} \rightarrow \mathrm{ZrO}_{2}(\mathrm{cr})+\mathrm{B}_{2} \mathrm{O}_{3}(\mathrm{l}), \quad T>\sim 873 \mathrm{~K} \\
& \mathrm{~B}_{2} \mathrm{O}_{3}(\mathrm{l}) \rightarrow \mathrm{B}_{2} \mathrm{O}_{3}(\mathrm{~g}), \quad T>\sim 1273 \mathrm{~K}
\end{aligned}
$$$$
\mathrm{ZrB}_{2}(\mathrm{cr})+(5 / 2) \mathrm{O}_{2} \rightarrow \mathrm{ZrO}_{2}(\mathrm{cr})+\mathrm{B}_{2} \mathrm{O}_{3}(\mathrm{~g}), \quad \mathrm{T}>\sim 2073 \mathrm{~K}
$$

根据 $\mathrm{ZrB}_{2}$ 在不同温度下的氧化产物二氧化锆 $\left(\mathrm{ZrO}_{2}\right)$ 与 $\mathrm{B}_{2} \mathrm{O}_{3}$ 的微观形貌 ${ }^{[11-12]}$, 大致可划分为 3 类 微观结构(图 1), 均与温度密切相关, 分别标识为低 温区间 $(<1273 \mathrm{~K})$, 中温区间(1273 2073 K) 和高温 区间 $(>2073 \mathrm{~K})$ 。

在低温有氧环境中, $\mathrm{ZrB}_{2}$ 氧化生成固态颗粒 $\mathrm{ZrO}_{2}$ 和液态 $\mathrm{B}_{2} \mathrm{O}_{3}$, 前者作为骨架, 具有较强的抗冲 刷能力; 后者作为骨架间的填充物及表面覆盖物,
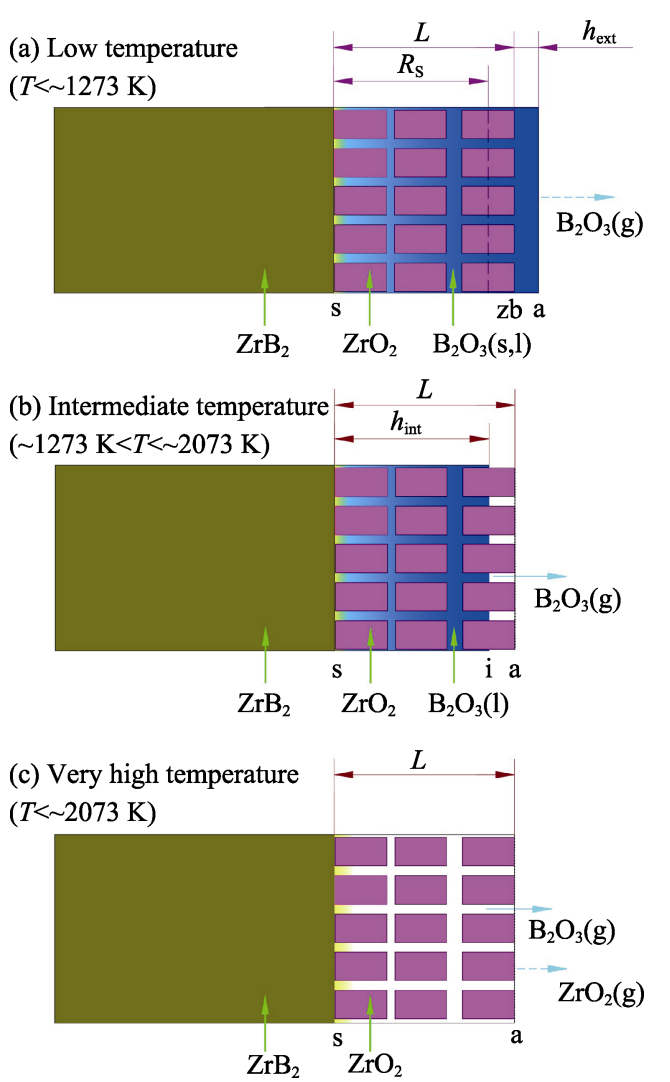

图 $1 \mathrm{ZrB}_{2}$ 氧化产物及其微观结构的示意图

Fig. 1 Schematic sketch of the oxidation products of $\mathrm{ZrB}_{2}$ and morphology assumed in the model

$L$ : scale thickness; $R_{\mathrm{s}}$ : recession of substrate; $h_{\mathrm{ext}}$ : outside layer thickness of $\mathrm{B}_{2} \mathrm{O}_{3} ; h_{\text {int }}$ : inside layer thickness of $\mathrm{B}_{2} \mathrm{O}_{3}$; a: interface between ambient and oxide; zb: interface between liquid and inside solid; $\mathrm{s}$ : interface between oxides and substrate; i: interface between inside liquid and solid

封闭了氧气通过孔隙快速到达原始材料界面的通 道。氧气要与底层基体材料反应, 需要首先通过 $\mathrm{ZrO}_{2}$ 和 $\mathrm{B}_{2} \mathrm{O}_{3}$ 组成的氧化膜, 因此相比物体表面浓 度边界层, 氧气在液态膜中的输运速率极低, 从而, 由扩散过程控制的氧化速率随之下降, 以此达到保 护底层材料的目的。当温度超过 $800 \mathrm{~K}$ 时, $\mathrm{ZrB}_{2}$ 在 大气环境中氧化过程明显 ${ }^{[13]}$, 而且表层玻璃态物质 $\mathrm{B}_{2} \mathrm{O}_{3}$ 的粘性减小, 流动性增强 ${ }^{[9]}$ 。当温度升高至 $1273 \mathrm{~K}$ 时, 表面的 $\mathrm{B}_{2} \mathrm{O}_{3}$ 蒸发显著, $\mathrm{B}_{2} \mathrm{O}_{3}$ 随温度升高 而逐渐退缩至 $\mathrm{ZrO}_{2}$ 孔隙中。而 $\mathrm{ZrO}_{2}$ 在温度从低到 高跨过其相变点 (相变温度约为 $1400 \mathrm{~K}$ )时, 会由单 斜晶系向四方晶系转变 ${ }^{[14]}$, 体积缩小约 $4 \%$ 。这种因 材料相变而发生的体积效应已在 $\mathrm{ZrB}_{2}-\mathrm{ZrO}_{2}$ 复相陶 瓷抗热震实验中被观察到 ${ }^{[4]}$, 并发现 $\mathrm{ZrB}_{2}$ 的氧化活 化能变化较大, 在 $1400 \mathrm{~K}$ 之上为 $322 \mathrm{~kJ} \cdot \mathrm{mol}^{-1}$, 之下 则小很多 ${ }^{[15]}$ 。当温度超过 $2073 \mathrm{~K}$ 时, $\mathrm{B}_{2} \mathrm{O}_{3}$ 的挥发速 率大于其生成速率, 而在氧化层几乎没有 $\mathrm{B}_{2} \mathrm{O}_{3}$, 仅 剩留多孔的 $\mathrm{ZrO}_{2}$ 。 $\mathrm{B}_{2} \mathrm{O}_{3}$ 的相对位置和存在形式可在 
模型的温度分区上加以区别, 而 $\mathrm{ZrO}_{2}$ 相变可在模型 的孔隙率上得到体现。

考虑到目前文献中没有明确给出 $\mathrm{ZrB}_{2}$ 的氧化 起始温度, 为方便研究, 本文根据现有氧化动力学 数据, 计算出 $\mathrm{ZrB}_{2}$ 的氧化起始温度位于 $660 \sim 742 \mathrm{~K}$ 之间。鉴于 $\mathrm{ZrB}_{2}$ 材料在高超声速领域中的使用温度 不超过 $1500 \mathrm{~K}$, 本文只介绍中低温区间模型。

\section{2 热化学反应模型}

\section{1 低温区间氧化模型}

在此温度区间, $\mathrm{ZrB}_{2}$ 氧化在材料表面形成氧化 膜。由于 $\mathrm{B}_{2} \mathrm{O}_{3}$ 的挥发性较低, 表层为液态 $\mathrm{B}_{2} \mathrm{O}_{3}$, 设 其厚度为 $h_{\text {ext。 }}$ 。下面一层为多孔结构的 $\mathrm{ZrO}_{2}$ 层, 设 其厚度为 $L$, 孔隙率(体积分数)为 $f$ 。设底层基材氧 化后退量为 $R_{\mathrm{S}}$ 。如图 1 所示, $\mathrm{B}_{2} \mathrm{O}_{3}$ 表层与外界环境 的界面标识为 $\mathrm{a} ; \mathrm{B}_{2} \mathrm{O}_{3}$ 与 $\mathrm{ZrO}_{2}$ 的界面标识为 $\mathrm{zb} ; \mathrm{ZrO}_{2}$ 与 $\mathrm{ZrB}_{2}$ 的界面标识为 $\mathrm{s}$ 。

氧气通过氧化膜向底层基材传输的流率表示为

$$
\begin{gathered}
J_{\mathrm{O}_{2}}=f \frac{\prod_{\mathrm{O}_{2}-\mathrm{B}_{2} \mathrm{O}_{3}}}{L}\left(p_{\mathrm{O}_{2}}^{\mathrm{zb}}-p_{\mathrm{O}_{2}}^{\mathrm{s}}\right)= \\
\frac{\prod_{\mathrm{O}_{2}-\mathrm{B}_{2} \mathrm{O}_{3}}}{h_{\mathrm{ext}}}\left(p_{\mathrm{O}_{2}}^{\mathrm{a}}-p_{\mathrm{O}_{2}}^{\mathrm{zb}}\right)
\end{gathered}
$$

式中, $J$ 为流率, $\mathrm{mol} \cdot \mathrm{m}^{-2} \cdot \mathrm{s}^{-1} ; L$ 为 $\mathrm{ZrO}_{2}$ 厚度, $\mathrm{m}$; $h_{\text {ext }}$ 为表层 $\mathrm{B}_{2} \mathrm{O}_{3}$ 厚度, $\mathrm{m} ; f$ 为孔隙率, 无量纲; $p_{\mathrm{O}_{2}}$ 为氧分压, $\mathrm{Pa}$ (本文中的压力单位均与此相同, 上标为位置标识); $\prod_{\mathrm{O}_{2}-\mathrm{B}_{2} \mathrm{O}_{3}}$ 为 $\mathrm{O}_{2}$ 在 $\mathrm{B}_{2} \mathrm{O}_{3}$ 液体中的 渗透系数, $\mathrm{mol} \cdot \mathrm{m}^{-1} \cdot \mathrm{s}^{-1} \cdot \mathrm{Pa}^{-1}$ 。借助试验数据 ${ }^{[16]}$, $\prod_{\mathrm{O}_{2}-\mathrm{B}_{2} \mathrm{O}_{3}}$ 表示为热力学温度 $(T, \mathrm{~K})$ 的关系:

$$
\Pi_{\mathrm{O}_{2}-\mathrm{B}_{2} \mathrm{O}_{3}}=0.109 \exp \left(-\frac{16724.83}{T}\right)
$$

底层 $\mathrm{ZrB}_{2}-\mathrm{B}_{2} \mathrm{O}_{3}$ 界面处的氧气分压可以根据反 应平衡常数计算 ${ }^{[17]}$.

$$
p_{\mathrm{O}_{2}}^{\mathrm{s}}=2.408 \times 10^{7} \exp \left(-\frac{94434.844}{T}\right)
$$

根据化学反应方程式与质量守恒, 可获得 $\mathrm{ZrO}_{2}$ 厚度增长方程:

$$
\frac{\mathrm{d} L}{\mathrm{~d} t}=\frac{1}{1-f} \frac{2}{5} J_{\mathrm{O}_{2}} \frac{M_{\mathrm{ZrO}_{2}}}{\rho_{\mathrm{ZrO}_{2}}}
$$

式中, $M$ 为摩尔质量, $\mathrm{kg} \cdot \mathrm{mol}^{-1}$ (下标表示组分种类); $\rho$ 为密度, $\mathrm{kg} \cdot \mathrm{m}^{-3} ; t$ 为时间, $\mathrm{s}$ 。

表层 $\mathrm{B}_{2} \mathrm{O}_{3}$ 的增加率表示为 $\Gamma_{\mathrm{B}_{2} \mathrm{O}_{3}}^{\mathrm{ext}}, \mathrm{mol} \cdot \mathrm{m}^{-2} \cdot \mathrm{s}^{-1}$, 它等于底层基材氧化生成率减去不断生长的多孔 $\mathrm{ZrO}_{2}$ 中的量以及表层挥发的量:

$$
\begin{gathered}
\Gamma_{\mathrm{B}_{2} \mathrm{O}_{3}}^{\mathrm{ext}}=\frac{\mathrm{d} n_{\mathrm{ext}, \mathrm{B}_{2} \mathrm{O}_{3}}}{\mathrm{~d} t}=\frac{\mathrm{d} L}{\mathrm{~d} t} \frac{1-f}{M_{\mathrm{ZrO}_{2}} / \rho_{\mathrm{ZrO}_{2}}}- \\
f \frac{\mathrm{d} L}{\mathrm{~d} t} \frac{\rho_{\mathrm{B}_{2} \mathrm{O}_{3}}}{M_{\mathrm{B}_{2} \mathrm{O}_{3}}}-J_{\text {evap }\left(\mathrm{B}_{2} \mathrm{O}_{3}\right)}
\end{gathered}
$$

当温度较低时, $\mathrm{B}_{2} \mathrm{O}_{3}$ 太黏而不能流动, 其表层 厚度为:

$$
\frac{\mathrm{d} h_{\mathrm{ext}}}{\mathrm{d} t}=\Gamma_{\mathrm{B}_{2} \mathrm{O}_{3}}^{\mathrm{ext}} \frac{M_{\mathrm{B}_{2} \mathrm{O}_{3}}}{\rho_{\mathrm{B}_{2} \mathrm{O}_{3}}}
$$

当温度超过 $800 \mathrm{~K}$ 时, $\mathrm{B}_{2} \mathrm{O}_{3}$ 流动性增强 ${ }^{[9]}$ 。由于 对比试验数据源自悬挂试样测试结果 ${ }^{[18]}, \mathrm{B}_{2} \mathrm{O}_{3}$ 的表 层厚度采用下式估算 ${ }^{[19]}$ :

$$
h_{\mathrm{ext}}=\left[\frac{3\left(M_{\mathrm{B}_{2} \mathrm{O}_{3}} \Gamma_{\mathrm{B}_{2} \mathrm{O}_{3}}^{\mathrm{ext}} l_{\text {spec }}\right) \mu_{\mathrm{B}_{2} \mathrm{O}_{3}}}{g \rho_{\mathrm{B}_{2} \mathrm{O}_{3}}^{2} \sin (\phi)}\right]^{1 / 3}
$$

式中, $l_{\mathrm{spec}}$ 为试样坚直方向的特征尺寸, $\mathrm{m} ; \mathrm{g}$ 为重 力加速度, $\mathrm{m} \cdot \mathrm{s}^{-2} ; \mu_{\mathrm{B}_{2} \mathrm{O}_{3}}$ 为 $\mathrm{B}_{2} \mathrm{O}_{3}$ 的粘性, $\mathrm{Pa} \cdot \mathrm{s} ; \phi$ 为 试样表面与水平面的夹角。

试样基材后退量 $R_{\mathrm{S}}([\mathrm{m}])$ 为:

$$
R_{\mathrm{s}}=L(1-f) \frac{M_{\mathrm{ZrB}_{2}} / \rho_{\mathrm{ZrB}_{2}}}{M_{\mathrm{ZrO}_{2}} / \rho_{\mathrm{ZrO}_{2}}}
$$

试样单位面积的质量变化为:

$$
\begin{aligned}
& \Delta W / A=L \rho_{\mathrm{ZrO}_{2}}(1-f)+ \\
& \quad\left(h_{\mathrm{ext}}+f L\right) \rho_{\mathrm{B}_{2} \mathrm{O}_{3}}-R_{\mathrm{s}} \rho_{\mathrm{ZrB}_{2}}
\end{aligned}
$$

式中, $\Delta W$ 为重量变化, $\mathrm{kg} \cdot \mathrm{s}^{-1} ; A$ 为面积, $\mathrm{m}^{2}$ 。

\section{2 中温区间氧化模型}

当温度高于 $1273 \mathrm{~K}$ 时, $\mathrm{B}_{2} \mathrm{O}_{3}$ 的挥发速率高于其 生成速率，此时 $\mathrm{B}_{2} \mathrm{O}_{3}$ 退缩至 $\mathrm{ZrO}_{2}$ 孔隙中，位置标识 为 $\mathrm{i}$, 设其厚度为 $h_{\mathrm{int}}$, 如图 1 所示。

根据反应的化学计量数, 可得中温区间 $\mathrm{ZrB}_{2}$ 的 氧化过程中各物质的关系:

$$
J_{\mathrm{O}_{2}}=\frac{5}{2} \dot{n}_{\mathrm{ZrO}_{2}}=\frac{5}{2} \dot{n}_{\mathrm{B}_{2} \mathrm{O}_{3}}
$$

式中, $\dot{\mathrm{n}}_{\mathrm{ZrO}_{2}} 、 \dot{n}_{\mathrm{B}_{2} \mathrm{O}_{3}}$ 分别表示氧化产物 $\mathrm{ZrO}_{2}$ 和 $\mathrm{B}_{2} \mathrm{O}_{3}$ 的生成速率, $\mathrm{mol} \cdot \mathrm{m}^{-2} \cdot \mathrm{s}^{-1}$ 。

在 $1300 \sim 3000 \mathrm{~K}$ 温度区间，利用液相、气相数 据 ${ }^{[20]}$, 可计算出 $\mathrm{B}_{2} \mathrm{O}_{3}$ 的蒸气压:

$$
p_{\mathrm{B}_{2} \mathrm{O}_{3}}^{\mathrm{i}}=1.841 \times 10^{8} \exp \left(-\frac{44661.247}{T}\right)
$$

气态 $\mathrm{B}_{2} \mathrm{O}_{3}$ 在孔隙“ $L-h_{\text {int }}$ ”段中的流率为

$$
J_{\mathrm{B}_{2} \mathrm{O}_{3}}=f D_{\mathrm{B}_{2} \mathrm{O}_{3}} \frac{C_{\mathrm{B}_{2} \mathrm{O}_{3}}-C_{\mathrm{B}_{2} \mathrm{O}_{3}}^{\mathrm{a}}}{L-h_{\mathrm{int}}}
$$

式中, $\mathrm{C}$ 为气态组分的摩尔浓度, $\mathrm{mol} \cdot \mathrm{m}^{-3}$ 。

生成的 $\mathrm{B}_{2} \mathrm{O}_{3}$ 减去挥发掉的气态 $\mathrm{B}_{2} \mathrm{O}_{3}$, 剩余的 
为孔隙中 $\mathrm{B}_{2} \mathrm{O}_{3}$ 的量:

$$
\Gamma_{\mathrm{B}_{2} \mathrm{O}_{3}}^{\mathrm{int}}=\dot{n}_{\mathrm{B}_{2} \mathrm{O}_{3}}-J_{\mathrm{B}_{2} \mathrm{O}_{3}}
$$

$\mathrm{B}_{2} \mathrm{O}_{3}$ 厚度变化为:

$$
\frac{\mathrm{d} h_{\text {int }}}{\mathrm{d} t}=\frac{1}{f} \Gamma_{\mathrm{B}_{2} \mathrm{O}_{3}}^{\mathrm{int}} \frac{M_{\mathrm{B}_{2} \mathrm{O}_{3}}}{\rho_{\mathrm{B}_{2} \mathrm{O}_{3}}}
$$

氧气首先在孔隙“ $L-h_{\text {inn }}$ ”段中扩散, 流率为:

$$
J_{\mathrm{O}_{2}}=f D_{\mathrm{O}_{2}} \frac{C_{\mathrm{O}_{2}}^{\mathrm{a}}-C_{\mathrm{O}_{2}}^{\mathrm{i}}}{L-h_{\mathrm{int}}}
$$

然后在液态 $\mathrm{B}_{2} \mathrm{O}_{3}$ 层中渗透, 氧气流率还可表示为:

$$
J_{\mathrm{O}_{2}}\left(\mathrm{~B}_{2} \mathrm{O}_{3}\right)=f \Pi_{\mathrm{O}_{2}-\mathrm{B}_{2} \mathrm{O}_{3}} \frac{p_{\mathrm{O}_{2}}^{\mathrm{i}}-p_{\mathrm{O}_{2}}^{\mathrm{s}}}{h_{\mathrm{int}}}
$$

$\mathrm{ZrO}_{2}$ 厚度增长方程为:

$$
\frac{\mathrm{d} L}{\mathrm{~d} t}=\left(\frac{1}{1-f}\right) \dot{n}_{\mathrm{ZrO}_{2}} \frac{M_{\mathrm{ZrO}_{2}}}{\rho_{\mathrm{ZrO}_{2}}}
$$

以上方程通过迭代算法求解。

底层基材后退量仍为式(8)。单位面积的质量变 化与式(9)类似:

$$
\Delta W / A=L \rho_{\mathrm{ZrO}_{2}}(1-f)+h_{\mathrm{int}} f \rho_{\mathrm{B}_{2} \mathrm{O}_{3}}-R_{\mathrm{s}} \rho_{\mathrm{ZrB}_{2}}
$$

\section{3 氧化起始温度}

Margrave 等 ${ }^{[15]}$ 研究 $\mathrm{ZrB}_{2}$ 的氧化过程中, 发现在 $1218 \sim 1529 \mathrm{~K}$ 温度范围和氧分压为 13375 和 $101325 \mathrm{~Pa}$ 时, 净重变化符合抛物型规律, 计算得到活化能为 $162.043 \mathrm{~kJ} \cdot \mathrm{mol}^{-1}$ 。以此条件下的氧化速率为参考, 降 低温度, 氧化速率下降, 至其千分之一时不再考虑 氧化。依据阿伦尼乌斯公式

$$
\ln \frac{k_{2}}{k_{1}}=-\frac{E_{a}}{R}\left(\frac{1}{T_{2}}-\frac{1}{T_{1}}\right)
$$

式中, $T_{2}$ 和 $T_{1}$ 分别对应高温、低温; $k_{2} / k_{1}$ 为反应速 率比。可得 $T_{1}=660 \sim 742 \mathrm{~K}$, 将此温度作为氧化的起 始温度。当温度超过 $773 \mathrm{~K}$ 时, 碳纤维的氧化比较 显著 ${ }^{[21]}$, 若碳基材料表面有 $\mathrm{ZrB}_{2}$ 涂层, 则氧气首先 与 $\mathrm{ZrB}_{2}$ 反应生成氧化膜, 降低了氧气通过氧化膜的 扩散速率，从而保护了底层的碳基材料。

\section{3 组分输运计算模型}

\section{1 孔隙中的气体扩散系数}

$\mathrm{ZrB}_{2}$ 在中高温下氧化, 其微观结构显示氧化层 存在大量极其微小的孔隙 ${ }^{[12]}$, 环境中的氧气可穿过 孔隙到达底层, 与 $\mathrm{ZrB}_{2}$ 反应, 生成的 $\mathrm{ZrO}_{2}$ 增厚氧化 层, 生成的气态 $\mathrm{B}_{2} \mathrm{O}_{3}$ 则通过孔隙向外逸出。对于扩 散过程控制的氧化行为, 需要确定气体组分的扩散, 主要包括分子扩散和 Knudsen 扩散。
某一组分在混合气体中由于分子浓度差造成的 扩散系数可近似表示为 ${ }^{[22]}$.

$$
\begin{aligned}
& D_{1,(2, \cdots, j)}=\frac{1}{\sum_{j}^{j \neq 1}\left(x_{j} / D_{1-j}\right)} \\
& x_{j}=\frac{n_{j}}{\sum_{k}^{k \neq 1} n_{k}}
\end{aligned}
$$

式中, $x_{j}$ 和 $n_{j}$ 分别为组分 $j$ 的摩尔分数和摩尔数; $D_{1,(2, \cdots, j)}$ 为组分 1 在混合气体中的扩散系数, $\left[\mathrm{m}^{2} \cdot \mathrm{s}^{-1}\right]$; $D_{1-j}$ 为组分 1 与组分 $j$ 组成的双组分扩散系数 ${ }^{[23]}$ :

$$
D_{1-2}=\frac{1.8583 \times 10^{-7} T^{3 / 2} \sqrt{\left(1 / M_{1}\right)+\left(1 / M_{2}\right)}}{\sigma_{12}^{2} \Omega_{\mathrm{D}} p}
$$

式中, $M_{1}$ 为组分 1 的相对分子量; $p$ 为混合气体的总 压力; $\sigma_{12}=0.5\left(\sigma_{1}+\sigma_{2}\right), \sigma$ 为碰撞直径, $\mathrm{nm} ; \Omega_{\mathrm{D}}$ 为碰撞对扩散的积分, 无量纲。 $\Omega_{\mathrm{D}}$ 与气体分子的分 子间作用力相关, 可由下式计算 ${ }^{[23]}$ :

$$
\begin{aligned}
\Omega_{\mathrm{D}}= & \frac{1.06036}{T^{* 0.15610}}+\frac{0.193000}{\exp \left(0.47635 T^{*}\right)}+ \\
& \frac{1.03587}{\exp \left(1.52996 T^{*}\right)}+\frac{1.76474}{\exp \left(3.89411 T^{*}\right)}
\end{aligned}
$$

式中, $T^{*}=k T / \varepsilon_{12}$ 为无量纲温度, $k$ 为玻尔兹曼常 数, $\varepsilon_{12}=\sqrt{\varepsilon_{1} \varepsilon_{2}}, \varepsilon$ 为分子间最大吸引能, $\mathrm{J}$ 。

氧化物 $\mathrm{ZrO}_{2}$ 中孔隙非常小, 因此气体在其中的 输运需要考虑 Knudsen 扩散效应:

$$
D_{\mathrm{K}}=\frac{4}{3}\left(\frac{8 R T}{\pi M}\right)^{1 / 2} \frac{r}{2}
$$

式中, $D_{\mathrm{K}}$ 为 Knudsen 扩散系数, $\mathrm{m}^{2} \cdot \mathrm{s}^{-1} ; M$ 为摩尔质 量; $r$ 为孔隙的等效半径, $m ; R$ 为通用气体常数。 气体最终有效扩散系数 $\left(D_{\text {eff }}\right)$ 由 Bosanquet 关系 确定 $^{[24]}$ :

$$
D_{\text {eff }}=\left(D_{\mathrm{K}}^{-1}+D_{1-2}^{-1}\right)^{-1}
$$

\section{2 物面组分蒸发流率}

高超声速飞行条件下气动加热严重, 防热材料 在有氧环境中出现化学及物理变化, 气体组分在近 壁面存在浓度梯度, 因此有必要考虑化学边界层里 组分的传输。组分包括环境中的 $\mathrm{O}_{2} 、 \mathrm{~N}_{2}$ 以及中高温 度下气态生成物 $\mathrm{B}_{2} \mathrm{O}_{3}$ 和 $\mathrm{ZrO}_{2}$ 。发生在物体表面的 组分 $j$ 的蒸发, 其蒸发流率可采用修正的 Langmuir 法则描述 ${ }^{[25]}$. 


$$
J_{\text {evap }, j}=\alpha \frac{p_{j}}{\left(2 \pi M_{j} R T\right)^{0.5}}
$$

式中, $M_{j}$ 为组分 $j$ 的摩尔质量; $p_{j}$ 为蒸气压; $\alpha$ 用 于描述浓度边界层有限扩散的影响, 其值与环境条 件有关。

\section{4 模拟结果分析}

当温度处于中温区间时, $\mathrm{B}_{2} \mathrm{O}_{3}$ 退缩至 $\mathrm{ZrO}_{2}$ 孔隙 中。图 2 为孔隙率对 $\mathrm{ZrB}_{2}$ 氧化的影响, 计算温度为 $1365 \mathrm{~K}$ 。由图可知, 随着孔隙率的增大, 氧化前后 凝聚相物质的单位面积质量变化也随之增大, 当孔 隙率为 0.1 与 0.001 时, 结果相差可达 13 倍左右。 由于孔隙的增多, 氧气渗透到底层基材的量也增加, 氧化加剧。由此可推断, 为了降低氧化程度, 一个 有效措施是在材料制备过程提高材料的致密性, 以降低材料反应生成表面氧化膜的过程中增加额 外孔隙率的几率。致密性也是材料制备的一个重要 参数 ${ }^{[4-5]}$ 。

为考察式(9)和式(18)所给出的模型对增重预测 的可靠性, 图 3 给出了模拟结果与试样测试结果的 对比。试验在加热炉中进行 ${ }^{[18]}, \mathrm{ZrB}_{2}$ 样品悬挂在天平 上, 炉中维持 $33335.925 \mathrm{~Pa}$ 的纯氧气氛, 气流速度为 $5 \times 10^{-3} \mathrm{~m} \cdot \mathrm{s}^{-1}$, 恒温加热。模拟结果发现, 温度从低到 高孔隙率分别选择 $0.06 、 0.04 、 0.02$ 时, 与试验结果 吻合较好。孔隙率是抗氧化膜的重要表征参数, 它 的确定依赖氧化产物 $\mathrm{ZrO}_{2}$ 与 $\mathrm{B}_{2} \mathrm{O}_{3}$ 的形态及二者的 物理作用, 可以通过氧化后的微观结构图采用统计 方法 ${ }^{[26]}$ 得到, 或定量金相技术 ${ }^{[27]}$ 和静力称重法 (hydrostatic weighing) ${ }^{[28]}$ 测量, 也可基于随机介质理 论进行建模研究 ${ }^{[29]}$ 。相对于等离子喷涂, $\mathrm{ZrB}_{2}$ 氧化 生成 $\mathrm{ZrO}_{2}$ 层, 其中的孔隙测量更难。直于试验技术, 本研究孔隙率通过查阅相关文献合理选择。低温下

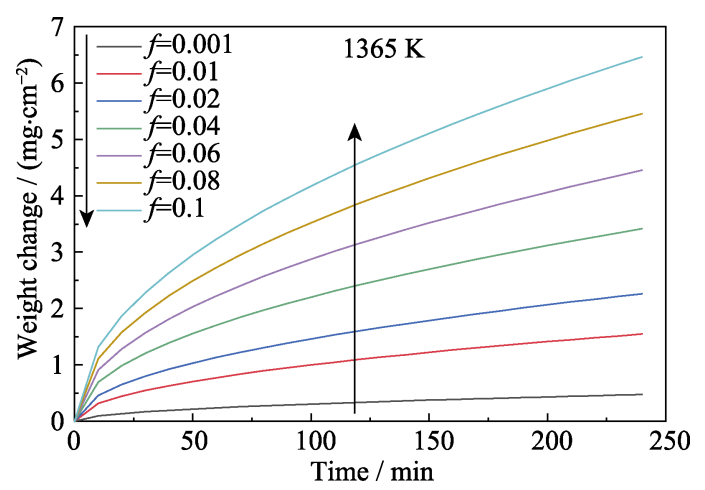

图 2 孔隙率对 $\mathrm{ZrB}_{2}$ 氧化前后质量损失的影响

Fig. 2 Effect of pore fraction on weight change of $\mathrm{ZrB}_{2}$
氧化产物 $\mathrm{B}_{2} \mathrm{O}_{3}$ 的挥发量小, 将孔隙率设为 0.06 。 当温度升高至 $1163 \mathrm{~K}$ 以上时, $\mathrm{B}_{2} \mathrm{O}_{3}$ 流动性增强, 对 生成的 $\mathrm{ZrO}_{2}$ 生长阻力变小, 此外需要开始考虑蒸 发, 孔隙率将随之减小, 直至 $\mathrm{ZrO}_{2}$ 相变点 $1400 \mathrm{~K}$, 孔隙率设为 0.04 。当温度超过相变点, $\mathrm{ZrO}_{2}$ 不再是 单斜晶系, 而是四方晶系, 孔隙率设为 0.02 。如等 离子喷涂 $\mathrm{ZrO}_{2}-4.9 \mathrm{~mol} \% \mathrm{Y}_{2} \mathrm{O}_{3}$ 粉末形成的涂层 ${ }^{[30]}$, 其孔隙率约为 $(14.2 \pm 0.6) \%$, 由此可以预测纯 $\mathrm{ZrO}_{2}$ 块 中孔隙率应更低。Parthasarathy 等 ${ }^{[8-10]}$ 研究了其取 值范围(0.025 0.2) 与氧化速率关系, 发现其取值 0.0015 和 0.03 时的模拟结果与试样氧化测试结果 接近。据此, 此处计算选取的孔隙率位于较为合理 的范围内。

在同一温度下，质量损失随时间变化呈现抛物 型。起始阶段，质量变化与时间呈现近似线性关系， 对应于氧化膜从无到有的初步形成，氧气扩散速率 相对较快; 很快, 质量变化与时间呈现抛物型关系, 对应于氧化膜全面形成，氧气扩散速率降低。随着 温度升高, 氧化速率加快, 质量损失增加。相对而 言, 当温度为 1098 与 $1163 \mathrm{~K}$ 时, 曲线变化较为缓 和，这是由于表面液态 $\mathrm{B}_{2} \mathrm{O}_{3}$ 的蒸发较小，氧化膜阻 碍氧气渗透的能力较强; 当温度为 $1263 \mathrm{~K}$ 时, 重量 增加量变化较大, 此时表面 $\mathrm{B}_{2} \mathrm{O}_{3}$ 挥发显著, 氧化膜 厚度变薄, 阻碍氧气渗透的能力有所下降; 而温度 为 $1365 、 1478 \mathrm{~K}$ 时，二者的重量变化较为接近，一 方面表面 $\mathrm{B}_{2} \mathrm{O}_{3}$ 液态层不复存在, 另一方面 $\mathrm{ZrO}_{2}$ 处 于相变点附近。此外, 在温度为 $1573 \mathrm{~K}$ 时, 材料重 量变化规律与其它几个温度下的变化规律有所差异, 这可能源于该温度下特殊的氧化行为, 如氧分压的 影响。McClaine ${ }^{[15]}$ 在关于高熔点金属的热力学和动 力学的总结报告里提到, Kuriakose 和 Margrave 在研 究 $\mathrm{ZrB}_{2}$ 的氧化过程中发现当温度为 $1329 \mathrm{~K}$ 、氧分压 在 13375 101325 Pa 之间时, 氧化速率与氧分压成线 性关系; 而当温度为 $1560 \mathrm{~K}$ 、氧分压在 1114.575 $5370.225 \mathrm{~Pa}$ 之间时，氧化速率与氧分压无关，此外， 这两个温度下氧化的活化能差异也很大。BerkowitzMattuck ${ }^{[11]}$ 也发现了类似规律。由于 $\mathrm{ZrB}_{2}$ 材料氧化 过程的复杂性, 本研究所建模型只选取了对整个氧 化过程有重要影响的关键参数, 未考虑在某些温度 下一些影响因素。

表 1 给出了 $\mathrm{B}_{2} \mathrm{O}_{3}$ 厚度与 $\mathrm{ZrO}_{2}$ 厚度之比。随着温 度升高, $\mathrm{B}_{2} \mathrm{O}_{3}$ 蒸发加剧, 厚度比减小, 基本呈 2 次方 下降。由此可知, 温度是影响氧化产物 $\mathrm{B}_{2} \mathrm{O}_{3}$ 和 $\mathrm{ZrO}_{2}$ 的生长厚度的重要因素, 进而间接影响氧气在液态 物质 $\mathrm{B}_{2} \mathrm{O}_{3}$ 中的溶解、渗透、扩散过程。为了降低材 料的氧化程度, 选择合适的使用温度, 确保材料表 

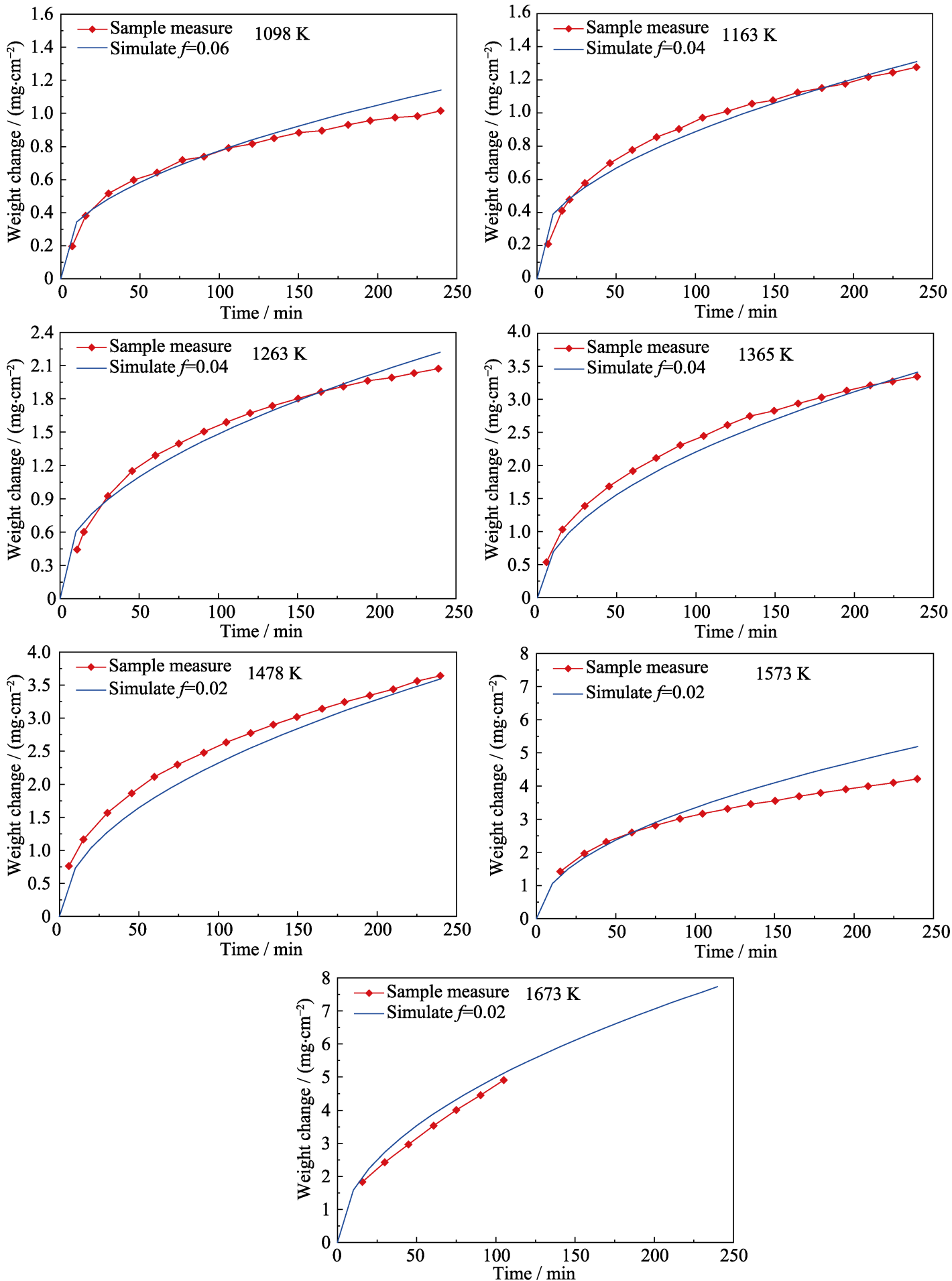

图 3 不同温度下 $\mathrm{ZrB}_{2}$ 氧化前后质量变化模拟结果与测试结果的比较

Fig. 3 Comparison of mass change obtained from simulation results and sample measurement results of $\mathrm{ZrB}_{2}$ at different temperatures

表 1 不同温度下氧化物 $\mathrm{B}_{2} \mathrm{O}_{3}$ 与 $\mathrm{ZrO}_{2}$ 的厚度比

Table 1 Ratio of thickness of $\mathrm{B}_{2} \mathrm{O}_{3}$ to that of $\mathrm{ZrO}_{2}$ at different temperatures

\begin{tabular}{cccccccc}
\hline$T / \mathrm{K}$ & 1098 & 1163 & 1263 & 1365 & 1478 & 1573 & 1673 \\
\hline Ratio & 1.05 & 1.04 & 1.03 & 0.99 & 0.96 & 0.87 & 0.70 \\
\hline
\end{tabular}

面维持一定厚度的 $\mathrm{B}_{2} \mathrm{O}_{3}$ 液态层, 将是一个不错的应 用选择。

\section{5 结论}

本研究基于改进的 $\mathrm{ZrB}_{2}$ 氧化模型得到如下结论:

1) 改进后的 $\mathrm{ZrB}_{2}$ 氧化模型能够较好地模拟加 热炉定温、恒氧分压条件下的 $\mathrm{ZrB}_{2}$ 氧化行为, 在中、 低温区间，模拟结果与试样测试结果吻合良好。模 
型的建立及有效性的验证将有助于未来进一步研究 变温条件下的氧化行为。

2) 孔隙对氧化过程有较大影响, 在相同的温 度、氧分压下, 孔隙率越大, 氧化程度越高。这意味 着在 $\mathrm{ZrB}_{2}$ 材料制备过程中, 提高材料致密性有助于 增强材料的抗氧化性能。

3) 当温度低于 $1273 \mathrm{~K}$ 时, 氧化产物 $\mathrm{B}_{2} \mathrm{O}_{3}$ 能在 $\mathrm{ZrB}_{2}$ 表面形成液态层并保持稳定。氧气首先溶解于 液态 $\mathrm{B}_{2} \mathrm{O}_{3}$ 中, 并渗透通过 $\mathrm{ZrO}_{2}$ 孔隙, 才能到达底部 基材。在此种状况下, 由扩散过程控制的氧化速率 极大地降低, 材料表现出最强的抗氧化性能。当 $\mathrm{B}_{2} \mathrm{O}_{3}$ 只存在于 $\mathrm{ZrO}_{2}$ 孔隙中的情况下, 材料的抗氧化 性能下降。

\section{参考文献:}

[1] CLOUGHeRTY E V, KALISH D, PETER E T. Research and development of refractory oxidation resistant diborides. AFML-TR-68$190,1968$.

[2] ZHANG GUO-JUN, LIU HAI-TAO, ZOU JI, et al. Chemical reactions in the life cycle of $\mathrm{ZrB}_{2}$ ceramics. Chinese Science Bulletin, 2015, 60(3): 276-286.

[3] LI GANG, HAN WEN-BO, JIANG JIU-XING. Mechanical properties and thermal shock resistance of $\mathrm{ZrB}_{2}$-SiC-AN ultrahigh temperature ceramics. Journal of Synthetic Crystals, 2009, 38, Special Edition: 36-39.

[4] SONG JIAN-RONG, LI JUN-GUO, SHEN QIANG, et al. Thermal shock and oxidation resistances of $\mathrm{ZrB}_{2}-\mathrm{ZrO}_{2}$ ceramics. Journal of the Chinese Ceramic Society, 2008, 36(5): 663-667.

[5] SONG JIE-GUANG, DU DA-MING, XU MING-HAN, et al. Oxidation behavior of $\mathrm{ZrB}_{2}$-matrix composite materials at high-temperature conditions. Powder Metallurgy Technology, 2015, 33(5): 336-340, 364.

[6] ZHOU HAI-JUN, ZHANG XIANG-YU, GAO LE, et al. Ablation properties of $\mathrm{ZrB}_{2}-\mathrm{SiC}$ ultra-high temperature ceramic coatings. Journal of Inorganic Materials, 2013, 28(3): 256-260.

[7] ZHAO HAI-LEI, WANG JIAN, LI WEN-CHAO. Study on oxidation kinetics of $\mathrm{ZrB}_{2}$-corundum-mullite composite. Naihuo Cailiao, 1998, 32(6): 322-325.

[8] PARTHASARATHY T A, RAPP R A, OPEKA M, et al. A model for the oxidation of $\mathrm{ZrB}_{2}, \mathrm{HfB}_{2}$ and $\mathrm{TiB}_{2}$. Acta Materialia, 2007, 55(17): 5999-6010.

[9] PARTHASARATHY T A, RAPP R A, OPEKA M, et al. A model for transitions in oxidation regimes of $\mathrm{ZrB}_{2}$. Materials Science Forum, 2008. 595-598: 823-832.

[10] PARTHASARATHY T A, RAPP R A, OPEKA M, et al. Effects of phase change and oxygen permeability in oxide scales on oxidation kinetics of $\mathrm{ZrB}_{2}$ and $\mathrm{HfB}_{2}$. Journal of the American Ceramic Society,
2009, 92(5): 1079-1086.

[11] BERKOWITZ-MATTUCK J B. High-temperature oxidation III. zirconium and hafnium diborides. Journal of the Electrochemical Society, 1966, 113(9): 908-914.

[12] HU PING, WANG GUO-LIN, WANG ZHI. Oxidation mechanism and resistance of $\mathrm{ZrB}_{2}-\mathrm{SiC}$ composites. Corrosion Science, 2009, 51(11): 2747-2732.

[13] ANDREEVA A F. Zirconium Diboride Low Resistance Layers. MAM'97-Materials for Advanced Metallization, Poster Session I, 1997.

[14] PAGE R J, SHORT R A, HALBACH C R. Evaluation of zirconia, thoria and zirconium diboride for advanced resistojet use. NASA CR-112075, 1972.

[15] MCCLAINE L A. Thermodynamic and kinetic studies for a refractory materials program, Part III. ASD-TDR-62-204 Part III, 1964.

[16] LUTHRA K L. Oxidation of carbon/carbon composites-a theoretical analysis. Carbon, 1988, 26(2): 217-224.

[17] CHASE JR M W. NIST-JANAF Thermochemical Tables $4^{\text {th }}$ Ed. Physical and Chemical Reference Data, Monograph No.9, 1998: 286, 291, 1756, 1780.

[18] TRIPP W C, GRAHAM H C. Thermogravimetric study of the oxidation of $\mathrm{ZrB}_{2}$ in the temperature range of 800 to $1500{ }^{\circ} \mathrm{C}$. Journal of the Electrochemical Society, 1971, 118(7): 1195-1199.

[19] PERRY R H, GREEN D W, MALONEY J O. Perry's Chemical Engineers' Handbook $7^{\text {th }}$ Ed.. New York: McGraw-Hill, 1997: 570.

[20] BARIN I. Thermochemical data of pure substances. New York: VCH Verlags-gesellschaft, 1995: 122-124.

[21] JACOBSON N S, CURRY D M. Oxidation microstructure studies of reinforced carbon/carbon. Carbon, 2006, 44(7): 1142-1150.

[22] WELTY J R, RORRER G L, FOSTER D G. Fundamentals of momentum, heat and mass transfer 6th Ed.. New York: WILEY, 2013, P448.

[23] BIRD R B, STEWART W E, LIGHTFOOT E N. Transport phenomena. New York: John Wiley, 2002: 526, 866.

[24] J. SZEKELY J, EVANS J SOHN W H Y. Gas Solid Reactions. New York: Academic Press, 1976: 25.

[25] DUFFA G. Ablative Thermal Protection System Modeling. Reston: AIAA, Inc. Press, 2012: 109.

[26] LI JIAN-FENG, ZHOU XIA-MING, DING CHUAN-XIAN. Statistical analysis of porosity variations in plasma sprayed $\mathrm{Cr}_{3} \mathrm{C}_{2}-$ $\mathrm{NiCr}$ coatings. Journal of Aeronautical Materials, 2000, 20(1): $33-38$.

[27] ZHANG HONG-SONG, WANG FU-CHI, MA ZHUANG, et al. Quantitative analysis of pores in plasma-sprayed $\mathrm{ZrO}_{2}$ coatings. Journal of Materials Engineering, 2006, Supplement 1: 407-425.

[28] PRATTEN N A. The precise measurement of the density in small samples. Materials Science, 1981, 16(7): 1737-1747.

[29] ZHAO YANG, LIN LI, MA ZHI-YUAN, et al. Establishing TBC random pore model based on random media theory. China Surface Engineering, 2010, 23(2): 78-81.

[30] FOX A C, CLYNE T W. Oxygen transport by gas permeation through the zirconia layer in plasma sprayed thermal barrier coating. Surface and Coatings Technology, 2004, 184(2/3): 311-321. 